\title{
COVID-19 Independent Risk Factors for Unfavorable Disease Progression: A Cross-Sectional Study from Romania
}

\author{
Ruxandra Laza ${ }^{1,2}$, Voichita Elena Lazureanu ${ }^{1,2}$, Virgil Filaret Musta ${ }^{1,2}$, Narcisa Daniela Nicolescu ${ }^{1,2}$, \\ Corina Vernic ${ }^{3}$, Anca Tudor $\mathbb{D}^{3}$, Roxana Critu ${ }^{4}$, Laura Vilceanu ${ }^{2}$, Roxana Paczeyka ${ }^{2}$, \\ Valerica Bica Profir ${ }^{2}$, Adelina Raluca Marinescu ${ }^{1,2,4}$, Alexandra Mocanu ${ }^{2,4}$, \\ Talida Georgiana Cut $\mathbb{D}^{1,2,4,5}$, Daniel Florin Lighezan ${ }^{6,7}$, Luminita Mirela Baditoiu ${ }^{8,9}$ \\ 'Department XIII, Discipline of Infectious Diseases, Victor Babes University of Medicine and Pharmacy, Timisoara, Romania; ${ }^{2}$ Clinical Hospital of \\ Infectious Diseases and Pneumophtisiology "Doctor Victor Babes", Timisoara, Romania; ${ }^{3}$ Department III, Discipline of Medical Informatics and \\ Biostatistics, Victor Babes University of Medicine and Pharmacy, Timisoara, Romania; ${ }^{4}$ Doctoral School, Victor Babes University of Medicine and \\ Pharmacy, Timisoara, Romania; ${ }^{5}$ Center for Ethics in Human Genetic Identifications, Victor Babes University of Medicine and Pharmacy, Timisoara, \\ Romania; ${ }^{6}$ Department of Internal Medicine I, Discipline of Medical Semiology I, Victor Babes University of Medicine and Pharmacy, Timisoara, \\ Romania; ${ }^{7}$ Municipal Emergency Hospital, Timisoara, Romania; ${ }^{8}$ Department XIII, Discipline of Epidemiology, Victor Babes University of Medicine and \\ Pharmacy, Timisoara, Romania; ${ }^{9}$ Multidisciplinary Research Center of Antimicrobial Resistance, Victor Babes University of Medicine and Pharmacy, \\ Timisoara, Romania
}

Correspondence: Talida Georgiana Cut, Victor Babes University of Medicine and Pharmacy, E. Murgu Square, Nr. 2, Timisoara, 30004I, Romania, Tel +40 755690250, Email talida.cut@umft.ro

Purpose: Since the initial documentation of severe acute respiratory syndrome coronavirus 2 (SARS-CoV-2) in late December 2019, the coronavirus disease 2019 (COVID-19) pandemic has become a leading cause of morbidity and mortality worldwide. Under these circumstances, the present study aimed to analyze the characteristics of hospitalized cases, according to the progression and severity of the disease, to reveal the main independent risk factors for death by COVID-19 in the western Romanian population.

Patients and Methods: This retrospective study included 936 patients admitted to Victor Babes Clinical Hospital of Infectious Diseases Timisoara, with COVID-19 between 1 February 2020 and 31 January 2021. The patients were divided into three subsamples based on disease severity: subsample I $(n=344)$, comprising patients with mild forms of the disease; subsample II $(n=475)$ comprising patients with moderate disease; and subsample III $(\mathrm{n}=117)$ for patients with severe disease. Biochemical analysis and assessment of disease severity were performed on all patients.

Results: The mean age was 48.76 years (two months to 94 years). The median values for age, number of hospitalization days, and disease duration days before hospital admission varied with high statistical significance between the three subsamples $(p<0.001)$. A statistically significant difference was also observed regarding body mass index (BMI), with higher values in subsample III $(p<$ $0.001)$. The in-hospital fatality rate was zero in subsample I, 3.58\% in subsample II, and $71.79 \%$ in subsample III $(p<0.001)$. Of the deceased patients, $69.31 \%$ were over the age of 65 years, $29.70 \%$ were aged 36-64 years, and $0.99 \%$ were aged 19-35 years. Cumulated comorbidities were recorded in $92.08 \%$ of the deceased patients.

Conclusion: Older age, the number of disease duration days before hospital admission, BMI, and renal pathology are independent risk factors for mortality in COVID-19 and can help clinicians to identify patients with poor prognosis and who are at high risk for mortality at an early stage.

Keywords: severe acute respiratory syndrome coronavirus 2, SARS-CoV-2, fatality rate, disease severity, risk factors

\section{Introduction}

The enveloped, positive-sense RNA single-stranded SARS-CoV-2, is a novel member of the $\beta$-coronavirus genus to infect humans since severe acute respiratory syndrome coronavirus (SARS-CoV) and Middle East Respiratory Syndrome 
Coronavirus (MERS-CoV) in the early 2000s. SARS-CoV-2 is the first pathogenic coronavirus to cause a large-scale outbreak with continuous strain on the global medical infrastructure and economics. ${ }^{1-3}$

The clinical spectrum of COVID-19 ranges from asymptomatic to bilateral pneumonia, consisting of extensive interstitial and alveolar inflammatory infiltrates, thickening of alveolar septa, vascular congestion, and lung oedema, often associated with acute respiratory distress syndrome (ARDS), respiratory failure, viral sepsis and multiorgan dysfunction. ${ }^{4-6}$ As a consequence of lung parenchymal damage and altered pulmonary circulation, pulmonary hypertension may develop, leading to right ventricular involvement and right-heart failure. ${ }^{7}$ Although respiratory pathology prevails, COVID-19 determines cardiovascular complications and is frequently responsible for a fatal outcome, especially in elderly patients with underlying cardiovascular diseases. The spectrum of cardiovascular complications is very large, ranging from sinus tachycardia or hypotension to various arrhythmias, myocardial ischemia, acute myocarditis with heart failure, or even pulmonary thromboembolism. ${ }^{8-12}$ By 10 May 2021, the COVID-19 there were 157,973,438 confirmed cases of COVID-19, with 3,288,455 deaths, representing the largest modern epidemiologic event after the Great Spanish Flu of 1918. ${ }^{13}$

In Romania, between 26 February 2020, when the first case was detected, and the end of 2020, 632,263 COVID-19 cases and 15,767 COVID-19 related-deaths were reported. Up until 10 May 2021, 1,066,731 cases and 29,034 COVID19 related-deaths were recorded nationwide. In Timiş County, throughout 2020, official records included 25,916 COVID19 cases and, respectively, 53,822, until 10 May 2021. ${ }^{14}$

Victor Babes Clinical Hospital of Infectious Diseases Timisoara, the largest specialized hospital in the West of Romania, admits mainly patients from Timis County, but also from the neighboring districts, and its Clinic II has 60 beds for infectious diseases, of which $80 \%$ are dedicated to COVID-19 patients. Under these circumstances, the present study analyzed the characteristics of hospitalized cases according to disease progression and severity to reveal the main independent COVID-19 risk factors for death in the western Romanian population.

\section{Materials and Methods}

This observational, cross-sectional, retrospective study comprised 936 patients admitted with COVID-19 to Clinic II of Victor Babes Clinical Hospital of Infectious Diseases Timisoara, between 1 February 2020 and 31 January 2021.

Patients who fulfilled the study criteria had a confirmed case of acute respiratory syndrome with the new coronavirus, ie, patients in whom the nucleic acid of SARS-CoV-2 was detected by a real-time reverse transcriptase polymerase chain reaction (RT-PCR) test via a nasopharyngeal or oropharyngeal swab and sputum or tracheobronchial exudate for severe cases. ${ }^{15}$ Treatment was conducted in accordance with the Romanian Ministry of Health's COVID-19 guidelines in force at the time of patient hospitalization. Based on disease severity, the patients were administered antiviral therapy (remdesivir or favipiravir), interleukin-1 receptor antagonists, humanized monoclonal antibodies against the interleukin-6 receptor, steroid treatment with dexamethasone or methylprednisolone, low molecular weight heparin, broadspectrum antibiotics, proton pump inhibitors, antitussives, or antipyretics. ${ }^{16-18}$ According to the Romanian National Institute of Public Health, clinical criteria include the presence of at least one of the following signs or symptoms: Cough, fever, shortness of breath, sudden onset of anosmia, ageusia or dysgeusia, in children - the presence of gastrointestinal symptoms (vomiting and accelerated intestinal transit), radiologic signs of COVID-19 compatible lesions, and epidemiologic criteria that include at least one of the followings:

- direct contact with a COVID-19 confirmed case, within 14 days before the date of symptom onset;

- residents or staff members in institutions caring for a vulnerable population, during 14 days before the date of onset, or institutions with confirmed SARS-CoV-2 transmission. ${ }^{18}$

Patients admitted to other departments, admitted for other diagnoses or discharged and readmitted, were excluded from the study. The following demographic and clinical data on SARS-CoV-2 infected patients were collected: period of hospitalization, clinical form upon admission, symptomatology, comorbidities, and status upon discharge.

\section{Sampling}

The 936 patients were classified into three subsamples depending on the clinical form of the infection and following the definitions of Romanian Public Health's COVID-19 guidelines. Thus: 


\section{Subsample I $(n=344)$}

Patients with a mild form of the disease:

oHistory of low-grade fever or afebrile upon admission;

oSymptoms of fatigue, dysphagia, irritative cough, smell, or taste disorders in the absence of imagistic signs of pulmonary lesions or organ dysfunction;

oNormal peripheral $\mathrm{O}_{2}$ saturation $\left(\mathrm{SpO}_{2}\right)$;

oInflammatory markers (C-reactive protein-CRP and fibrinogen) within normal or slightly elevated limits;

oAbsent coagulation disorders;

oNormal platelet count;

oNormal lymphocyte count.

\section{Subsample II $(n=475)$}

Patients with a moderate form of the disease having at least one of the followings:

oFever and signs of non-severe pneumonia;

oLocalized or minor pulmonary lesions affecting $<50 \%$ of the total pulmonary area;

oPossible organ damage;

$\mathrm{oSpO}_{2}$ between $90 \%$ and $95 \%$ with no oxygen therapy required;

oModerate lymphopenia;

oIncreased inflammatory syndrome ( $\mathrm{CPR}>20 \mathrm{mg} / \mathrm{dL}$ and fibrinogen $\leq 1464 \mathrm{mg} \%$ ).

\section{Subsample III $(n=117)$}

Patients with signs of severe pneumonia having at least one of the followings:

oDyspnea;

oRespiratory rate $\geq 30 / \mathrm{min}$;

oSpO2 $\leq 90 \%$ at rest;

oArterial oxygen partial pressure $\left(\mathrm{PaO}_{2}\right) /$ fraction of inspired oxygen $\left(\mathrm{FiO}_{2}\right) \leq 300 \mathrm{mmHg}$;

oLow hemoglobin;

oSevere lymphopenia;

oIncreased lactate (over two times the normal level);

oCRP over $100 \mathrm{mg} / \mathrm{dL}$;

oCoagulation disorders with platelet counts below $100,000 / \mathrm{mm}^{3}$ and increased D-dimers;

oOrgan lesion signs;

oImagistic aspects - extended lesions $>50 \%$, ground glass lesions, progression of lesions with $50 \%$ or more within 24-48 hours. ${ }^{19}$

\section{Molecular Method for Case Certification}

COVID-19 confirmation was carried out using the CFX-96 Real-Time System IVD (Bio-Rad, California, USA). The viral RNA was extracted with the NIMBUS extractor, using the STARMag $96 \times 4$ Universal Cartridge Kit (Seegene, Seoul, South Korea) and amplified with the Allplex 2019-nCoV Kit (Seegene, Seoul, South Korea), which amplifies three viral targets and one control gene: RdRP gene (Cal Red 610); N gene (Quasar 670); E gene (FAM); Internal control (HEX). According to the World Health Organization's (WHO) recommendations, 45 amplification cycles were used. Because the CFX-96 Real-Time System IVD used in this study expresses only the threshold cycle (CT) values (the limit of detection is 100 copies/reaction) and does not provide the quantitative value of the viral load, the samples were labeled as positive when specific SARS-CoV-2 genes were detected.

\section{Definitions}

Body mass index (BMI; $\mathrm{kg} / \mathrm{m}^{2}$ ) was calculated according to the following formula: BMI $=$ weight $(\mathrm{kg}) \div$ height $\mathrm{t}^{2}\left(\mathrm{~m}^{2}\right)$. Obesity was defined as a BMI over $30.0 \mathrm{~kg} / \mathrm{m}^{2}$ (for both genders) and morbid obesity as values over $40.0 \mathrm{~kg} / \mathrm{m}^{2}{ }^{20}$ 
Excess in-hospital mortality was calculated by the difference between the number of deceased patients in the department between February and December 2019 to the total reported number of patients admitted to the Department during the same period and the same report calculated for the same time interval in $2020 .{ }^{21}$ The COVID-19 fatality ratio was calculated by reporting the number of patients who died by this pathology to the number of cases admitted to the clinic with the respective pathology during the period of 1 February to 31 December $2020 .^{22}$ Lethality was represented by the percentage of deaths by COVID-19 to the total number of deaths recorded in the clinic during the mentioned period.

Data collection respected and protected confidentiality. The study was conducted in accordance with the Declaration of Helsinki and was approved by the Ethics Commission of SCBIPVBT, no. 4534/14.05.2021. Due to the retrospective study design, informed consent could not be obtained from the patients.

\section{Statistical Analysis}

Data analysis was conducted using IBM SPSS Statistics 20 software (SPSS Inc., Chicago, USA). Continuous variables are characterized by the median and interquartile (IQR), while categorical variables are characterized by the value and percentage. Data distribution was tested by the Kolmogorov-Smirnov test. Numerical variables were compared by Kruskal-Wallis for all the three subsamples, a Mann-Whitney $U$-test was used for comparison between two subsamples, while for nominal ones, the chi-squared test was used (Fisher's exact test). For bivariate correlations, we applied Spearman correlation. A risk factor was defined in the case of OR/RR $>1$ (lower limit of $95 \% \mathrm{CI}>1$ ), under statistical significance conditions. Variables that met the risk factor criteria under univariate analysis (OR $>1$ (lower limit of $95 \%$ $\mathrm{CI}>1$ )) were investigated by logistic regression. The model was chosen according to the Nagelkerke $\mathrm{R}^{2}$ coefficient and the Hosmer-Lemeshow test to assess the deviation from the theoretical model. All the statistical tests were two-tailed, and the statistical significance threshold $(p)$ was set at 0.05 .

\section{Results}

The age of the 936 COVID-19-positive cases hospitalized in Clinic II of Victor Babes Clinical Hospital of Infectious Diseases Timisoara, varied between two months and 94 years, with a mean value of 48.76 years (mean value for subsample $\mathrm{I}=31.15$, standard deviation $=20.24$; mean value for subsample II $=57.01$, standard deviation $=13.78$; mean value for subsample III $=67.12$, standard deviation $=13.99$ ). No significant differences between three subsamples were observed regarding gender distribution $(\mathrm{F} / \mathrm{M}=49.89 / 50.11 \% p=0.926)$. Patients came mainly from urban areas $(\mathrm{U} /$ $\mathrm{R}=64.74 / 35.26 \%, p<0.001)$.

The distribution of cases showed a first peak in April, caused by imported cases, a significant decrease, due to the physical distancing imposed during the state of emergency (declared in Romania between 15 March 2020 and 15 May 2020) followed by the multiplication of cases in parallel with the increase in community transmission. Fatalities reached a peak in November 2020 (Figure 1). The global fatality by COVID-19 during the period 1 February 2020 to 31 January 2021 was $10.79 \%$, and lethality was $92.66 \%$. The in-hospital mortality rate in the clinic and during the above-mentioned period reached $8.25 \%$, with an excess mortality of $7.11 \%$ compared to the 1 February to 31 January 2020 period (when it was $1.14 \%)$.

Most hospitalized cases presented medium-severity clinical forms $(50.75 \%, n=475)$, while $36.75 \%$, $(n=344)$ were mild cases and $12.50 \%,(n=117)$ were severe.

The analysis of the three subsamples, grouped according to the clinical form of the disease-the result of the conflict between SARS-CoV-2 and the power of the host immune defense, is presented in Table 1.

The median values for age, number of hospitalization days, and number of disease duration days before hospital admission varied with high statistical significance between the three subsamples $(p<0.001)$. A statistically significant difference was observed among the three subsamples regarding body mass index, with higher values in subsample III $(p<0.001)$. Gender differences were also highly statistically significant, with the predominance of women in the sample having mild forms, while men were predominant in the medium and severe samples $(p<0.001)$ (Table 1$)$.

The disease progression differed with high statistical significance between the three subsamples. The in-hospital fatality rate was zero in subsample I, $3.58 \%$ in subsample II, and $71.79 \%$ in subsample III $(p<0.001)$. Of the patients with 


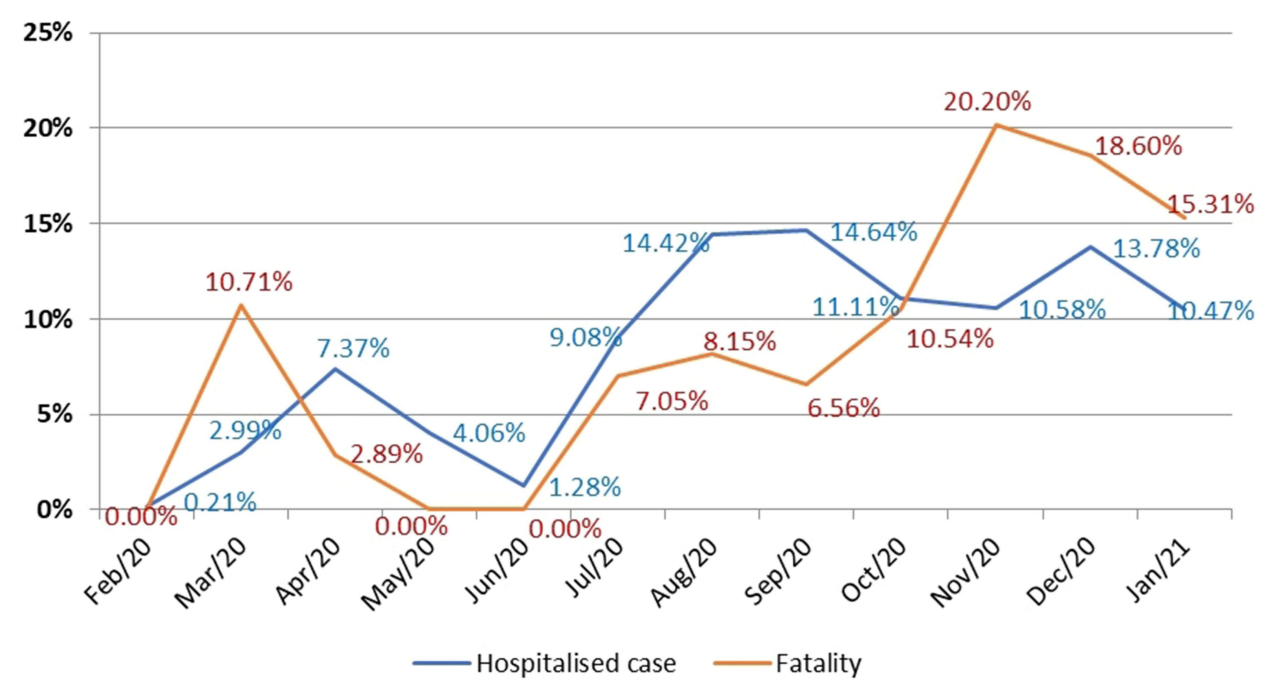

Figure I Distribution of COVID-19 hospitalized cases and the case fatality ratio $(N=936)$.

negative disease progression, 57 (56.43\%) died during hospitalization in the intensive care unit (ICU), $89.47 \%$ of whom required orotracheal intubation. Comorbidities varied with high statistical significance, reaching $33.43 \%$ in mild cases, $75.58 \%$ in medium cases, and $88.89 \%$ in severe cases. Meanwhile, cardiovascular pathology (high blood pressure, ischemic cardiomyopathy, and atrial fibrillation), diabetes, obesity, and kidney diseases varied with high statistical significance between the three subsamples; in contrast, pulmonary pathology and oncologic diseases did not vary ( $p=0.259$ and 0.430 , respectively). Moreover, no statistical significance was observed regarding autoimmune diseases ( $p=0.473)$, chronic liver diseases $(p=0.120)$, organ transplantation $(p=0.706)$ and pregnancy $(p=0.075)$ between the three subsamples. No patient included in the study had human immunodeficiency virus (HIV). Additionally, statistically significant differences were observed in neuropsychic and gastric diseases between patients with different clinical forms. Of note, $71.79 \%$ of severe cases presented cardiovascular comorbidities and $29.06 \%$ had diabetes mellitus (Table 1).

Intricate aspects of symptom onset were noticed. All known respiratory, digestive, neurological, nonspecific, and atypical variants were found. Of the neurological symptoms, anosmia, ageusia and asthenia were recorded in moderate/ severe cases, as well as in mild cases.

Clinical severity correlated significantly with age (strong correlation; rho $=0.621, p<0.001$ ), as well as the number of disease duration days prior to hospital admission and the BMI (medium correlation; rho $=0.424 / 0.454, p<0.001$ ).

The analysis of risk factors for death by COVID-19, using multivariate logistic regression, identified age, the number of disease days prior to hospital admission, BMI, and renal pathology as independent risk factors, as shown in Table 2.

Meanwhile, the mild and moderate clinical forms represent the usual disease progression of SARS-CoV-2 infection, and the severe clinical form often belongs to unfavorable disease progression followed by death: $-81.19 \%$ of the deceased patients had a severe clinical form. Death occurred in $18.81 \%$ of the patients with the moderate form and in none of those with mild clinical forms. Of the deceased patients, $69.31 \%$ were over the age of 65 , 29.70\% were 36-64 years, and $0.99 \%$ were $19-35$ years. Cumulated comorbidities were recorded in $92.08 \%$ of the deceased (Table 2).

\section{Discussion}

The mean age of the 936 COVID-19 patients hospitalized in the clinic was 48.76 years, which was similar to that in a 2020 Chinese study that received national coverage, in which the mean age of 1590 patients was 48.9 years. ${ }^{23}$ In Romania, a multicentric study on a smaller sample of 432 patients published in December 2020 identified a mean age of 68 years, but the subjects came from 36 districts of Romania, while the present study mainly focused on the four districts in western Romania. Most of the patients came from Timiș, but others came from Arad, Caraș-Severin or Hunedoara. ${ }^{24}$ In a study conducted in China at the beginning of the pandemic, and published in March 2020 in The Lancet, the median age was 56 years for hospitalized patients. ${ }^{25}$ The mean age of samples may vary, influenced by the life expectancy of the 
Table I Statistical Analysis of the Three Subsamples $(N=936)$

\begin{tabular}{|c|c|c|c|c|}
\hline Variable & $\begin{array}{l}\text { Ss I Mild Form } \\
\qquad n I=344\end{array}$ & $\begin{array}{l}\text { Ss II Medium Form } \\
\qquad n 2=475\end{array}$ & $\begin{array}{l}\text { Ss III Severe Form } \\
\qquad n 3=117\end{array}$ & $p^{\text {sig }}$ \\
\hline Age median [IQR] & $\begin{array}{c}30.00[15.00- \\
45.00]\end{array}$ & $58.00[48.00-67.00]$ & $69.00[57.00-78.00]$ & $<0.001^{\mathrm{s}}$ \\
\hline No. of hospitalization days median [IQR] & $7.00[3.00-11.00]$ & $10.00[7.00-14.00]$ & $11.00[4.00-16.00]$ & $<0.001^{\mathrm{s}}$ \\
\hline No. of previous days of disease median [IQR] & $2.00[0.00-4.00]$ & $4.00[3.00-6.00]$ & $6.00[4.00-7.00]$ & $<0.001^{\mathrm{s}}$ \\
\hline Body mass index median [IQR] & $22.85[19.08-25.66]$ & $26.06[23.88-29.30]$ & $29.07[25.69-34.48]$ & $0.025^{\mathrm{s}}$ \\
\hline Female (n, \%) & $208(60.47)$ & $211(44.42)$ & $48(41.03)$ & \multirow[t]{2}{*}{$<0.001^{\mathrm{s}}$} \\
\hline Male (n, \%) & $136(39.53)$ & $264(55.58)$ & $69(58.97)$ & \\
\hline Treatment for COVID-19 prior to hospitalization (n, \%) & $47(13.66)$ & $102(21.47)$ & $30(25.64 \%)$ & $0.003^{s}$ \\
\hline \multicolumn{5}{|c|}{ Disease progression } \\
\hline Cured (n, \%) & $176(51.16)$ & $264(55.58)$ & $20(17.09)$ & $<0.001^{\mathrm{s}}$ \\
\hline Discharged upon request $(\mathrm{n}, \%)$ & $157(45.64)$ & $180(37.89)$ & $7(5.98)$ & $<0.001^{\mathrm{s}}$ \\
\hline Death $(n, \%)$ & $0(0)$ & $17(3.58)$ & $84(71.79)$ & $<0.001^{\mathrm{s}}$ \\
\hline Transfer due to late complications ( $\mathrm{n}, \%)$ & I (0.29) & $5(1.05)$ & $3(2.56)$ & 0.089 ins \\
\hline Intensive care unit transfer (n, \%) & $0(0)$ & $3(0.63)$ & $3(2.56)$ & $0.011^{\mathrm{s}}$ \\
\hline \multicolumn{5}{|c|}{ Comorbidities } \\
\hline None $(n, \%)$ & $229(66.57)$ & $116(24.42)$ & $13(11.11)$ & $<0.001^{\mathrm{s}}$ \\
\hline Pulmonary (n, \%) & $18(5.23)$ & $34(7.16)$ & II (9.40) & 0.259 ins \\
\hline Cardiovascular (n, \%) & $46(13.37)$ & 261 (54.95) & 84 (7I.79) & $<0.00 \mathrm{I}^{\mathrm{s}}$ \\
\hline Diabetes (n, \%) & $12(3.49)$ & $100(21.05)$ & $34(29.06)$ & $<0.001^{\mathrm{s}}$ \\
\hline Obesity (n, \%) & $19(5.52)$ & $101(21.26)$ & $49(41.88)$ & $<0.001^{\mathrm{s}}$ \\
\hline Neoplasms (n, \%) & $16(4.65)$ & $29(6.10)$ & $9(7.69)$ & 0.430 ins \\
\hline Neuropsychic (n, \%) & $19(5.52)$ & $39(8.21)$ & $18(15.38)$ & $0.003^{s}$ \\
\hline Gastroenterological (n, \%) & $9(2.62)$ & $33(6.95)$ & $7(5.98)$ & $0.001^{\mathrm{s}}$ \\
\hline Renal (n, \%) & $5(1.45)$ & $25(5.26)$ & $18(15.38)$ & $<0.001^{\mathrm{s}}$ \\
\hline Autoimmune diseases* $(n, \%)$ & $6(1.74)$ & $14(2,95)$ & $2(I .7 I)$ & 0.473 ins \\
\hline Chronic liver diseases ( $n, \%)$ & $3(0.87)$ & $14(2,95)$ & $3(2.56)$ & 0.120 ins \\
\hline Organ transplantation $(\mathrm{n}, \%)$ & I (0.29) & $3(0.63)$ & I (0.85) & 0.706 ins \\
\hline Pregnancy (n, \%) & $3(0.87)$ & $0(0)$ & $0(0)$ & 0.075 ins \\
\hline \multicolumn{5}{|c|}{ Symptom onset } \\
\hline Atypical (n, \%) & $3(0.87)$ & $9(1.89)$ & $4(3.42)$ & 0.168 ins \\
\hline Respiratory (n, \%) & $125(36.34)$ & $388(81.68)$ & II 15 (98.29) & $<0.001^{\mathrm{s}}$ \\
\hline Digestive (n, \%) & $47(13.66)$ & $106(22.32)$ & $13(\mid \mathrm{I} .1 \mathrm{I})$ & $<0.001^{\mathrm{s}}$ \\
\hline Febrile (n, \%) & $124(36.05)$ & $287(60.42)$ & $59(50.43)$ & $<0.001^{\mathrm{s}}$ \\
\hline
\end{tabular}


Table I (Continued).

\begin{tabular}{|c|c|c|c|c|}
\hline Variable & $\begin{array}{c}\text { Ss I Mild Form } \\
n \mid=344\end{array}$ & $\begin{array}{l}\text { Ss II Medium Form } \\
\qquad n 2=475\end{array}$ & $\begin{array}{l}\text { Ss III Severe Form } \\
\qquad n 3=117\end{array}$ & $p^{\text {sig }}$ \\
\hline Neurological (n, \%) & $107(31.10)$ & $199(41.89)$ & $19(16.24)$ & $<0.001^{\mathrm{s}}$ \\
\hline Nonspecific (n, \%) & $36(10.46)$ & $196(41.26)$ & $52(44.44)$ & $<0.001^{\mathrm{s}}$ \\
\hline Asymptomatic (n, \%) & $107(31.10)$ & $12(2.53)$ & $2(I .7 I)$ & $<0.001^{s}$ \\
\hline
\end{tabular}

Note: *Includes autoimmune thyroiditis, systemic lupus erythematosus, psoriasis, rheumatoid arthritis, ankylosing spondylitis, systemic scleroderma, polymyositis, and dermatomyositis.

Abbreviations: ${ }^{\text {sig }}$, significance; ${ }^{\text {s, }}$ significant difference; ${ }^{\text {ins }}$, insignificant difference; $N$, number; Ss, subsample.

population from which the sample comes or by the pandemic control measures in the respective territory. A lower number of mild clinical forms in our study were noted in the 0-16 years age group, ie, 109 patients (11.64\%), which may be explained either by a better nonspecific natural immune defense (involving also pattern recognition receptors (PRRs) and pathogen-associated molecular patterns (PAMPs) receptors) or by a higher involvement of the angiotensin-converting enzyme 2 (ACE2) circulating form, having a protective role. ${ }^{26}$ The ACE2 enzyme, which has been proven to be the main cell receptor for SARS-CoV-2, has some peculiarities: Although it is well expressed in the digestive tract, gallbladder, heart, kidney, and seminal glands, SARS-CoV-2 more frequently causes pulmonary lesions. Even if the tissue expression of ACE2 allows the virus to enter the cell, the soluble form of ACE2 behaves like a protective factor against COVID-19. Thus, the activity of circulating ACE2 is weak in overweight or hypertensive patients but stronger in children; the fact that ACE2 activity positively correlates with estrogen expression supports the observation that there might be a relatively higher protection of children to adults and of women to men. ${ }^{27}$

Regarding the deceased patients in the present study, the median age was significantly higher than in surviving discharged patients (70 versus 50), similar to the value found in the study conducted by Fei Zhou et al, where the median age of deceased cases was $69 .{ }^{25}$ In a study of 355 deceased patients in Italy, a population with a life expectancy higher than in Romania's, the mean age was 78.5 years. ${ }^{28}$ These two studies, which included patients from China and Italy, two countries with very high mortality rates, found similar results to our study, and concluded that old age is a risk factor for patient mortality from COVID-19.

The gender distribution was similar ( $\mathrm{F} / \mathrm{M}=49.89 \% / 50.11 \%)$ among patients, but with a predominance of women in mild and men in medium and severe clinical forms $(p<0.001)$. Gender was close to the statistical significance threshold $(p=0.058)$, but it was not identified as a risk factor for death following univariate analysis. The fact that, in most cases, women presented mild/medium forms of disease while the majority of severe forms occurred in men is linked more to the viral load at the entry gate, the quality of nonspecific immune defense, the expression of ACE2 receptors, and the protective role of the soluble ACE2 in women; supplemented by the protective role of estrogen. ${ }^{29}$ This relative protection for women is not supported by the statistical data in the present study, regarding the gender distribution of deaths, because individual peculiarities and cumulated comorbidities come first. Still, the latest analysis of risk factors for death in confirmed COVID-19 cases, elaborated by the National Institute of Public Health in Romania in November 2020, identified being male as a risk factor $(\mathrm{OR}=1.67,95 \% \mathrm{CI} 1.48-1.88){ }^{30}$

The predominance of urban residents in the case of the patients hospitalized with SARS-CoV-2 infection (U/R=64.74/ $35.26 \%, p<0.001)$ is explained by the airborne transmission favored by urban agglomeration, cohabitation in small and crowded spaces, sometimes with suboptimal sanitation, and air pollution significantly higher than in rural areas. A study elaborated by the collective led by Conticini et al hypothesized that exposure to highly polluted air for long periods leads to a weakening of the defense mechanisms in the respiratory tract. They revealed a correlation between the Air Quality Index (which measures the level of pollution) and the number of COVID-19 deaths in regions of Lombardy and Emilia Romagna, where there was no direct proportional decrease in cases after a decrease in pollution after the pandemic. This shows that pollution has long-term effects on the respiratory system that cannot be rapidly reversed. ${ }^{31}$ 
Table 2 Risk Factors for Death by COVID-19 $(N=936)$

\begin{tabular}{|c|c|c|c|c|c|c|}
\hline \multirow[t]{2}{*}{ Variable } & \multirow{2}{*}{$\begin{array}{l}\text { Subsample Discharged } \\
\text { Patients } n I=835\end{array}$} & \multirow{2}{*}{$\begin{array}{c}\text { Subsample Deceased } \\
\text { Patients n2 }=101\end{array}$} & \multicolumn{2}{|c|}{ Univariate Analysis } & \multicolumn{2}{|c|}{ Multivariate Analysis } \\
\hline & & & $p^{\text {sig }}$ & OR $[95 \% \mathrm{Cl}]$ & $p^{\text {sig }}$ & aOR $[95 \% \mathrm{Cl}]$ \\
\hline Age (median, [IQR]) & $50[33.00-63.00]$ & $70[60.00-80.00]$ & $<0.001^{\mathrm{s}}$ & $1.08[1.06-1.10]$ & $<0.001^{\mathrm{s}}$ & $1.09[1.07-1.11]$ \\
\hline No. of hospitalization days (median, [IQR]) & $9[5.00-14.00]$ & $8[4.00-15.00]$ & $0.364^{\text {ins }}$ & $1.01[0.98-1.05]$ & l & I \\
\hline $\begin{array}{l}\text { No. of disease duration days before hospital } \\
\text { admission (median, [IQR]) }\end{array}$ & $4[2.00-3.00]$ & $5[3.00-7.00]$ & $<0.001^{\mathrm{s}}$ & $1.19[1.12-1.27]$ & $<0.001^{\mathrm{s}}$ & $1.15[1.07-1.24]$ \\
\hline Body mass index (median [IQR]) & $24.97[22.32-27.78]$ & $27.68[24.77-33.64]$ & $<0.001^{\mathrm{s}}$ & $1.09[1.06-1.13]$ & $<0.001^{\mathrm{s}}$ & $1.08[1.04-1.13]$ \\
\hline Female (n, \%) & $426(51.02)$ & $4 \mathrm{I}(40.59)$ & \multirow[t]{2}{*}{$0.058^{\text {ins }}$} & \multirow[t]{2}{*}{$0.66[0.42-1.02]$} & \multirow[t]{2}{*}{ l } & \multirow[t]{2}{*}{ l } \\
\hline Male (n, \%) & $409(48.98)$ & $60(59.41)$ & & & & \\
\hline Cardiovascular pathology (n, \%) & $353(42.27)$ & $38(37.72)$ & $0.370^{\text {ins }}$ & $0.82[0.53-1.29]$ & I & I \\
\hline Chronic renal pathology (n, \%) & $27(3.23)$ & $21(20.79)$ & $<0.001^{\mathrm{s}}$ & $7.86[4.07-15.15]$ & $<0.001^{\mathrm{s}}$ & $6.65[3.25-13.61]$ \\
\hline Diabetes (n, \%) & 117 (14.0I) & $29(28.7 \mathrm{I})$ & $<0.001^{\mathrm{s}}$ & $2.47[1.50-4.07]$ & l & I \\
\hline
\end{tabular}

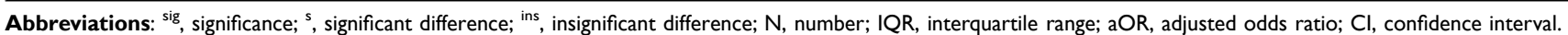


The percentage of severe forms of the total number of hospitalized cases in this retrospective study was $12.50 \%$, slightly lower than the $16 \%$ identified by one of the Chinese studies mentioned previously. ${ }^{27}$

COVID-19 led to a 7.23-fold increase in in-hospital mortality in the surveyed department, and the case fatality ratio by this infectious disease reached $10.79 \%$ in the context of a global national mean (outpatient and inpatient) of $2.75 \% .{ }^{32}$ In a study conducted by Wei-Jie Guan et al, fatality among hospitalized patients was $3.1 \%$ during the pandemic onset in totally different populations and intensity of epidemiological restrictions. ${ }^{25}$ The increasing fatality curve may be explained by the fact that the period from the onset of symptoms and the addressability to tertiary medical units increased during the pandemic to the point where medium and severe cases that did not improve under outpatient treatment became predominant.

In Romania, specific challenges were faced with regard to managing the spread and limiting the impact of the disease, ranging from healthcare infrastructure to demographic and sociocultural aspects. As the country has a sizeable diaspora, major difficulties were faced when large numbers of individuals from highly affected areas returned to Romania. ${ }^{33}$ The fast implementation of control measures successfully averted a surge in the number of COVID-19 cases during the initial phases of the epidemic, and by early May, the increase in incidence in Romania was comparable to that of moderately affected countries. As such, on 15 May 2020, the authorities replaced the national state of emergency with a state of alert, thereby relaxing most of the restrictions. ${ }^{33,34}$ Despite the drastic measures taken by the authorities, public confusion emerged in the following stages of the outbreak, as a result of misinformation regarding COVID-19. This increased the possibility of a worse outcome for vulnerable populations; thus, the highest fatality recorded for our study was in November 2020 to December 2020, a period with a relatively low hospitalization rate.

This study revealed that severe clinical forms had a significantly higher median number of days of disease duration prior to hospital admission compared to the mild and medium ones. In addition, the percentage of patients with ambulatory treatment prior to hospitalization was significantly higher for the medium and severe clinical forms ( $11.36 \%$ mild vs $21.47 \%$ medium and $25.64 \%, p=0.003$ ), which with hospitalization delay, drew attention to the importance of evaluation and rapid admission, in order to maximize the chances of favorable outcome. The ambulatory treatment included antipyretics, antitussives, vitamins $\mathrm{C}$ and D, and zinc. Azithromycin was also sometimes prescribed in the first half of 2020, while for those with mild forms, an oral anticoagulant (INN apixaban) was sometimes replaced by antiplatelet medication. Corticosteroids in anti-inflammatory doses were also recommended from October 2020.

In the entire sample $(N=936)$, comorbidities were dominated by cardiovascular pathology in $41.77 \%$ of patients, but no major cardiovascular diseases-symptomatic coronary heart disease, arrhythmia, or heart failure-were observed. Cardiovascular pathology was not identified as a death risk factor after applying univariate analysis $(\mathrm{OR}=0.82[0.53-$ 1.29 ], $p=0.370$ ), but more as a consequence of aging.

Type 2 diabetes mellitus (T2DM) was present in $15.59 \%$ of patients, $29.06 \%$ in those with severe forms and in 29 deceased patients (28.71\%). Because of impaired insulin secretion due to beta-pancreatic cell dysfunction combined with increased insulin resistance in peripheral tissues (muscle and fat tissue), chronic hyperglycemia was present, which is difficult to manage and can cause microvascular (cardiac, renal, and neurological) or macrovascular (cardiac, cerebral, and peripheral) complications demonstrating that the long disease progression of diabetes mellitus adds an important risk factor to SARS-CoV-2 infection. ${ }^{35,36}$ In this study sample, T2DM, as an associated disease, was identified as a risk factor for unfavorable disease progression only in the univariate analysis ( $\mathrm{OR}=2.47$ [1.50-4.07], $p<0.001)$, with a bidirectional effect - T2DM aggravated COVID-19, which, in turn, aggravated T2DM.

Obesity is associated with increased mortality. The death risk of all causes is $50 \%-100 \%$ higher as compared to normal-weight individuals, largely because of cardiovascular impairment. Hyperinsulinemia and insulin resistance are the usual indicators. In both women and men, obesity is an independent risk factor for ischemic cardiomyopathy, myocardial infarction, and heart failure. Obesity, especially of the abdominal variety, is associated with an atherogenic lipid profile having increased levels of LDL-cholesterol, very low-density lipoproteins, and triglycerides, and low levels of HDLcholesterol and adiponectin, an adipokine with vascular protector and anti-inflammatory roles. An extended mass of fat tissue is associated with increased levels of pro-inflammatory cytokines. The macrophages derived from fat tissue might be the primary source of local and systemic pro-inflammatory cytokines. ${ }^{35,36}$ 
In our study, $18.05 \%$ of the patients (5.52\% of those with the mild form of COVID-19, $21.26 \%$ with the moderate form, and $41.88 \%$ with the severe form) had obesity as the main comorbidity $(p<0.001)$. A BMI over $40 \mathrm{~kg} / \mathrm{m}^{2}$, which means morbid obesity, was identified in two patients $(0.58 \%)$ of subsample I, 15 patients $(3.16 \%)$ of subsample II and 11 patients $(9.40 \%)$ of subsample III $(p<0.001)$. Among the deceased patients, 34 (33.66\%) were obese, of whom seven $(6.93 \%)$ had morbid obesity, which meant that the obesity diagnosis represented a death risk factor $(\mathrm{OR}=2.63[1.63-$ 4.23], $p<0.001)$, as did morbid obesity ( $\mathrm{OR}=2.89$ [1.01-7.27], $p=0.024)$ in the univariate analysis. The multivariate logistic regression model identified BMI as an independent risk factor of death $(\mathrm{aOR}=1.08$ [1.04-1.13], $p<0.001)$.

Renal pathology plays an important role in the disease progression of COVID-19. Knowing that ACE2 expression is well represented in the kidney, nephron lesions (glomeruli, proximal tubule, Henle's loop and distal tubule) are expected to occur, beginning with the first phases of infection, leading to an early and marked disturbance of the fluid electrolyte and acid-base balance. ${ }^{37}$ In this study, $5.13 \%$ of all patients presented with renal pathology and 21 of the deceased $(20.79 \%)$ had chronic renal diseases, the criteria for independent death risk factors being met ( $\mathrm{aOR}=6.65$ [3.25-13.61], $p<0.001)$.

In the analysis of death risk factors in confirmed COVID-19 cases elaborated by the National Institute of Public Health in Romania, cardiovascular diseases were first ( $\mathrm{OR}=3.48$ [3.06-3.97]), followed by chronic renal diseases ( $\mathrm{OR}=2.91$ [2.39-3.53]) and oncologic diseases $(\mathrm{OR}=2.58$ [1.99-3.34]). Diabetes was sixth ( $\mathrm{OR}=2.05$ [1.75-2.39]), after chronic hepatic and pulmonary diseases. ${ }^{30}$ In our research, neither pulmonary pathology (6.73\% of the total number of patients) nor neoplastic diseases $(5.77 \%)$ significantly varied among subsamples, while neuropsychic pathology prevailed in those with severe forms compared to the other subsamples $(p=0.003)$.

Compared to the study conducted by Fei Zhou et al, cardiovascular and renal comorbidities were more frequent (41.77 vs $38 \%$, and 5.13 vs $1 \%$, respectively), whereas diabetes prevailed in the Asian study (15.59 vs 19\%), in the context of a higher prevalence of this pathology in Southeast Asia. ${ }^{25,38}$

In $96.04 \%$ of the deceased patients in this study, respiratory symptoms were predominant (dry cough, dyspnea, and low oxygen saturation). In 63 patients in whom dyspnea became extreme (marked suffocation sensation), medical surveillance continued in the ICU for prosthetic respiratory treatment and supportive mechanical ventilation. Of these, 57 (56.43\%) died during hospitalization in the ICU, $89.47 \%$ of whom required orotracheal intubation.

In the present study, unfavorable disease progression was directly related to the degree of pulmonary infiltration, together with hypoxia, hypercapnia, and organ dysfunction with subsequent onset. An acute onset, marked by wellexpressed respiratory symptoms, a computed tomography (CT) evaluation performed as timely as possible, will improve the chances for better control of the disease progression. As pulmonary hyperinflammation is equivalent to a procoagulation status, and clinical surveillance imposes a permanent control over the following parameters: oxygen saturation, coagulation processes, fibrinogen and fibrin degradation products, dynamics of blood sugar levels, fluidelectrolyte, and acid-base imbalances.

The results of this study provide evidence of the importance not only of endogenous factors at risk of adverse outcomes (age, associated pathologies, such as overweight, or kidney disease) but also the need for rapid addressability immediately after the onset of symptoms in the tertiary medical system for evaluation and hospitalization. Prolonging the ambulatory period, sometimes even with treatment, may be a risk factor for worsening COVID-19 infection. This aspect is important because after a year and a half of the pandemic, social and medical disparities have deepened, and the increased level of anxiety of patients has generated a tendency to avoid contact with the medical system. ${ }^{39}$ Moreover, the unfavorable disease progression of COVID-19 in this study was in direct relation to the extended pulmonary inflammatory process. Evidence of direct involvement of the SARS-CoV-2 virus is illustrated by the deaths of relatively young people with no significant pathological history, which leaves open the possibility of reevaluating the claim that the virus does not kill healthy people who have no comorbidities.

\section{Study Limitations}

This retrospective, analytical study involved a single center, so the results cannot be generalized. Furthermore, despite the significant number of cases, the minimal necessary data did not allow for elaboration of a wider multivariate analysis model. The study did not allow the analysis of direct biological values (CRP or lactate-dehydrogenase) as potential risk 
factors. Therefore, this study constitutes the base for a future case-control study, focused on analyzing the involvement of paraclinical and therapeutic aspects in disease progression. Smoking, although it has a wide involvement in cardiovascular and respiratory pathology, was not included as an independent risk factor due to the practical difficulty of adding accurate information in a retrospective study on the number of cigarettes smoked per day and smoking duration in years.

\section{Conclusion}

This study delimitated a disease progression framework, certain risk factors and clinical observations requiring further research. The SARS-CoV-2 infection generated an important in-hospital mortality excess, and fatalities reached $10.79 \%$. The multivariate logistic regression model identified age, number of disease duration days before hospital admission, BMI, and renal pathology as independent death risk factors for COVID-19. The aspect of delayed treatment potentially leading to unfavorable outcomes was especially relevant as the increased level of anxiety in patients in the course of the pandemic generated a tendency to avoid contact with the medical system. We noted the potential for inducing negative disease progression in the case of chronic metabolic and renal pathologies, while cardiovascular diseases proved to be a consequence of aging rather than a death risk factor.

\section{Disclosure}

The authors declare no conflicts of interest in this work.

\section{References}

1. Coronaviridae Study Group of the International Committee on Taxonomy of Viruses. The Species Severe acute respiratory syndrome-related coronavirus: classifying 2019-nCoV and naming it SARS-CoV-2. Nat Microbiol. 2020;5:536-544. doi:10.1038/s41564-020-0695-Z

2. Laza R, Musta VF, Nicolescu ND, et al. Cutaneous Manifestations in SARS-CoV-2 Infection-A Series of Cases from the Largest Infectious Diseases Hospital in Western Romania. Healthcare. 2021;9(7):800. doi:10.3390/healthcare9070800

3. Enache A, Ciocan V, Muresan CO, et al. Postmortem Documentation of SARS-CoV-2 in Utero and Postpartum Transmission, through Amniotic Fluid, Placental, and Pulmonary Tissue RT-PCR. Appl Sci. 2021;11(20):9505. doi:10.3390/app11209505

4. Zhu N, Zhang D, Wang W, et al. A Novel Coronavirus from Patients with Pneumonia in China, 2019. N Engl J Med. 2020;382:727-733. doi:10.1056/NEJMoa2001017

5. Cut TG, Tudoran C, Lazureanu VE, et al. Spontaneous Pneumomediastinum, Pneumothorax, Pneumopericardium and Subcutaneous EmphysemaNot So Uncommon Complications in Patients with COVID-19 Pulmonary Infection-A Series of Cases. J. Clin. Med. 2021;10(7):1346. doi: $10.3390 / \mathrm{jcm} 10071346$

6. Marinescu AR, Laza R, Musta VF, et al. Clostridium Difficile and COVID-19: general Data, Ribotype, Clinical Form, Treatment-Our Experience from the Largest Infectious Diseases Hospital in Western Romania. Medicina. 2021;57(10):1099. doi:10.3390/medicina57101099

7. Tudoran C, Tudoran M, Lazureanu VE, et al. Evidence of Pulmonary Hypertension after SARS-CoV-2 Infection in Subjects without Previous Significant Cardiovascular Pathology. Journal of Clinical Medicine. 2021;10(2):199. doi:10.3390/jcm10020199

8. Tudoran M, Tudoran C, Lazureanu V, et al. Alterations of Left Ventricular Function Persisting during Post-Acute COVID-19 in Subjects without Previously Diagnosed Cardiovascular Pathology. JPM. 2021;11(3):225. doi:10.3390/jpm11030225

9. Longhitano Y, Racca F, Zanza C, et al. Venous Thrombo-Embolism in Hospitalized SARS-CoV-2 Patients Treated with Three Different Anticoagulation Protocols: prospective Observational Study. Biology. 2020;9(10):310. doi:10.3390/biology9100310

10. Yancy CW, Fonarow GC. Coronavirus Disease 2019 (COVID-19) and the Heart-Is Heart Failure the Next Chapter? JAMA Cardiol. 2020;5 (11): 1216. doi:10.1001/jamacardio.2020.3575

11. Hu H, Ma F, Wei X, Fang Y. Coronavirus fulminant myocarditis treated with glucocorticoid and human immunoglobulin. Eur Heart J. 2021;42 (2):206. doi:10.1093/eurheartj/ehaa190

12. Xiong T-Y, Redwood S, Prendergast B, Chen M. Coronaviruses and the cardiovascular system: acute and long-term implications. Eur Heart J. 2020;41(19):1798-1800. doi:10.1093/eurheartj/ehaa231

13. World Health Organization. Coronavirus (COVID-19) Dashboard. Available from: https://covid19.who.int/. Accessed February 16, 2022.

14. Evolution of COVID-19 in Romania Dashboard. Available from: https://www.graphs.ro/. Accessed February 16, 2022.

15. World Health Organization. WHO COVID-19: case definitions: updated in public health surveillance for COVID-19, published 16 December 2020; 2020. Available from: https://apps.who.int/iris/handle/10665/337834. Accessed February 16, 2022.

16. National Institute of Public Health, The Coronavirus Disease 2019 (COVID-19) Treatment Guidelines. Available from: http://legislatie.just.ro/ Public/DetaliiDocument/229019. Accessed February 16, 2022.

17. National Institute of Public Health. The Coronavirus Disease 2019 (COVID-19) Treatment Guidelines. Available from: https://www.cnscbt.ro/ index.php/informatii-pentru-personalul-medico-sanitar/1525-metodologia-de-supraveghere-a-covid-19-actualizare-16-03-2020/file. Accessed February 16, 2022.

18. National Institute of Public Health, The COVID-19 case definitions. Available from: http://www.cnscbt.ro/index.php/2031-definitii-de-caz-sirecomandari-de-prioritizare-a-testarii-pentru-covid-19-actualizare-14-10-2020-1. Accessed February 16, 2022.

19. Son KB, Lee T, Hwang S. Disease severity classification and COVID-19 outcomes, Republic Of Korea. Bull World Health Organ. 2021;99(1):6266. doi:10.2471/BLT.20.257758 
20. Zierle-Ghosh A, Jan A. Physiology, Body Mass Index. StatPearls. StatPearls Publishing; 2021. Available from: http://www.ncbi.nlm.nih.gov/ books/NBK535456/. Accessed November 17, 2021.

21. Nucera G, Chirico F, Raffaelli V, Marino P. Current challenges in COVID-19 diagnosis: a narrative review and implications for clinical practice. Ital J Med. 2021;15:129-134. doi:10.4081/itjm.2021.1474

22. Chirico F, Nucera G, Magnavita N. Estimating case fatality ratio during COVID-19 epidemics: pitfalls and alternatives. J Infect Dev Ctries. 2020;14(5):438-439. doi:10.3855/jidc. 12787

23. Guan W, Liang W, Zhao Y, et al. Comorbidity and its impact on 1590 patients with COVID-19 in China: a nationwide analysis. Eur Respir J. 2020;55(5):2000547. doi:10.1183/13993003.00547-2020

24. Pantea Stoian A, Pricop-Jeckstadt M, Pana A, et al. Death by SARS-CoV 2: a Romanian COVID-19 multi-centre comorbidity study. Sci Rep. 2020;10(1):21613. doi:10.1038/s41598-020-78575-w

25. Zhou F, Yu T, Du R, et al. Clinical course and risk factors for mortality of adult inpatients with COVID-19 in Wuhan, China: a retrospective cohort study. Lancet. 2020;395(10229):1054-1062. doi:10.1016/S0140-6736(20)30566-3

26. Vaduganathan M, Vardeny O, Michel T, McMurray JJV, Pfeffer MA, Solomon SD. Renin-Angiotensin-Aldosterone System Inhibitors in Patients with Covid-19. N Engl J Med. 2020;382(17):1653-1659. doi:10.1056/NEJMsr2005760

27. Reynolds HR, Adhikari S, Pulgarin C, et al. Renin-Angiotensin-Aldosterone System Inhibitors and Risk of Covid-19. $N$ Engl J Med. 2020;382 (25):2441-2448. doi:10.1056/NEJMoa2008975

28. Onder G, Rezza G, Brusaferro S. Case-Fatality Rate and Characteristics of Patients Dying in Relation to COVID-19 in Italy. JAMA. 2020. doi:10.1001/jama.2020.4683

29. Stilhano RS, Costa AJ, Nishino MS, et al. SARS-CoV-2 and the possible connection to ERs, ACE2, and RAGE: focus on susceptibility factors. FASEB j. 2020;34(11):14103-14119. doi:10.1096/fj.202001394RR

30. National Institute of Public Health, Epidemiological analysis (2) risk factors for death with COVID 19. Available from: http://www.cnscbt.ro/index. php/analiza-cazuri-confirmate-covid19/2064-analiza-epidemiologica-2-factori-de-risc-pentru-deces-cu-covid-19. Accessed February 16, 2022.

31. Conticini E, Frediani B, Caro D. Can atmospheric pollution be considered a co-factor in extremely high level of SARS-CoV-2 lethality in Northern Italy? Environ Pollut. 2020;261:114465. doi:10.1016/j.envpol.2020.114465

32. National Institute of Public Health, Distribution by counties of cases confirmed with COVID-19 in Romania. Available from: https://instnsp.maps. arcgis.com/apps/opsdashboard/index.html\#/5eced796595b4ee585bcdba03e30c127. Accessed February 16, 2022.

33. Dascalu S. The Successes and Failures of the Initial COVID-19 Pandemic Response in Romania. Front Public Health. 2020;8:344. doi:10.3389/ fpubh.2020.00344

34. Coronavirus Cases. Worldometer (2020). Available from: https:/www.worldometers.info/coronavirus/. Accessed February 16, 2022.

35. Caussy C, Pattou F, Wallet F, et al. Prevalence of obesity among adult inpatients with COVID-19 in France. Lancet Diabetes Endocrinol. 2020;8 (7):562-564. doi:10.1016/S2213-8587(20)30160-1

36. Lim S, Bae JH, Kwon HS, Nauck MA. COVID-19 and diabetes mellitus: from pathophysiology to clinical management. Nat Rev Endocrinol. 2021;17(1):11-30. doi:10.1038/s41574-020-00435-4

37. Hardenberg JB, Luft FC. Covid-19, ACE2 and the kidney. Acta Physiol. 2020;230:1. doi:10.1111/apha.13539

38. Rhee EJ. Diabetes in Asians. Endocrinol Metab. 2015;30(3):263. doi:10.3803/EnM.2015.30.3.263

39. Dehelean L, Papava I, Musat MI, et al. Coping Strategies and Stress Related Disorders in Patients with COVID-19. Brain Sci. 2021;11(10):1287. doi:10.3390/brainsci11101287

\section{Publish your work in this journal}

The International Journal of General Medicine is an international, peer-reviewed open-access journal that focuses on general and internal medicine, pathogenesis, epidemiology, diagnosis, monitoring and treatment protocols. The journal is characterized by the rapid reporting of reviews, original research and clinical studies across all disease areas. The manuscript management system is completely online and includes a very quick and fair peer-review system, which is all easy to use. Visit http://www.dovepress.com/testimonials.php to read real quotes from published authors.

Submit your manuscript here: https://www.dovepress.com/international-journal-of-general-medicine-journal 